\title{
FDA approves Eli Lilly's baricitinib
}

The FDA approved Eli Lilly and Incyte's baricitinib for rheumatoid arthritis (RA), making it the third JAK inhibitor to hit the market.

Lilly first filed the immunosuppressive drug for FDA review in 2016, but the agency rejected an approval and requested additional data to determine appropriate dosing levels and to better characterize safety concerns. The sponsors resubmitted the drug in 2017, and the agency has now approved a low-dose regimen of the drug, with a boxed warning noting the risks of serious infection, malignancy and thrombosis. Analysts forecast sales of US\$1.3 billion by 2024 , show Cortellis consensus sales forecasts.

JAK family proteins mediate the effects of a wide range of cytokines, modulating T cell function, B cell function, haematopoiesis and more. Baricitinib, as well as Lilly's first-in-class JAK inhibitor ruxolitinib and Pfizer's second-to-market tofacitinib, are non-selective JAK inhibitors that can downregulate the entirety of the JAK family signalling spectrum. While a few companies are developing more pan-JAK inhibitors, many drug developers are now focusing on next-generation selective JAK inhibitors that will only block specific JAK signalling cascades. AbbVie's upadacitinib and Gilead Sciences and Galapagos's filgotinib are selective JAK1 inhibitors, and are in phase III trials for RA and Crohn's disease, amongst other indications. Pfizer's PF-06651600 acts on JAK3, and is in phase II trials for RA, Crohn's disease, ulcerative colitis and hair loss. And Bristol-Myers Squibb’s BMS-986165 inhibits the TYK2 member of the JAK family, and is in phase II trials for psoriasis and systemic lupus erythematosus.

A recent review of the field in Nature Reviews Drug Discovery cautioned that important questions remain about the benefits of improved JAK selectivity, optimal routes and dosing regimens and how to identify patients who will respond to these drugs. "Although many next-generation [JAK inhibitors] are reasonably selective in vitro, their selectivity in the clinical arena is not as fully characterized and remains to be proved. Moreover, current studies indicate that selective and pan-[JAK inhibitors] are equally effective for rheumatoid arthritis, but the same may not be true for other immune-mediated diseases," the authors write.

Asher Mullard

\section{mRNA vaccines get another booster}

Sanofi partnered with Translate Bio in a deal worth up to US $\$ 800$ million to develop mRNA vaccines against up to five infectious disease pathogens.

mRNA vaccines are an emerging new vaccine modality in which patients are treated with mRNA oligonucleotides that encode either an antigen of interest or an antigen as well its viral replication machinery. Although their application has been limited by the instability and inefficient in vivo delivery of mRNA, a recent review in Nature Reviews Drug Discovery summarized how technological advances have now largely overcome these issues.

"Currently, mRNA vaccines are experiencing a burst in basic and clinical research. The past 2 years alone have witnessed the publication of dozens of preclinical and clinical reports showing the efficacy of these platforms. Whereas the majority of early work in mRNA vaccines focused on cancer applications, a number of recent reports have demonstrated the potency and versatility of mRNA to protect against a wide variety of infectious pathogens," the authors write. The authors also caution that two recent trials found that immunogenicity in humans may be more modest than anticipated and side effects can be non-trivial with at least some mRNA vaccine platforms.

Translate Bio, formerly called RaNA Therapeutics, and Sanofi have not yet disclosed what pathogens they will be working on. But several companies have advanced infectious disease mRNA vaccines into the clinic. Argos Therapeutics and partner Kyowa Hakko Kirin are testing AGS-004 in phase II HIV trials, and Curevac is testing its CV7201 in a phase I rabies trial. Moderna's mRNA-1388, mRNA-1851, mRNA-1325, mRNA-1647 and mRNA-1653 are in phase I trials for chikungunya virus, influenza, zika virus, cytomegalovirus, human parainfluenza virus and human metapneumovirus, respectively.

Companies are also working on mRNA cancer vaccines that train the immune system to find and kill cancer cells, and on mRNA-based therapeutics that can replace or supplement aberrant proteins.

Asher Mullard

\section{Ebola outbreak prompts experimental drug rollout}

With Ebola flaring up once again, the Democratic Republic of the Congo (DRC) has authorized the experimental use of five treatments for infected patients. These are Mapp Biopharmaceutical's ZMapp, Regeneron's REGN-EB3, the National Institute of Allergy and Infectious Diseases' mAb114, Gilead Sciences' remdesivir and MediVector's favipiravir.

ZMapp consists of a cocktail of three monoclonal antibodies (mAbs) that target separate glycoprotein epitopes on the Ebola viral envelope to inactivate the virus. It yielded promising signs of benefit when trialled in the 2014-2015 Ebola outbreak in West Africa, but this trial did not reach its enrolment goals and failed to hit its prespecified statistical threshold for efficacy. REGN-EB3 is also a cocktail of three mAbs that bind the glycoprotein. It has promising efficacy in animal models of disease, and has completed a phase I safety study. mAb114 binds the glycoprotein as well. It entered into phase I safety trials earlier this year.

Remdesivir is a nucleoside analogue that blocks Ebola's RNA polymerase to prevent viral replication. It was used successfully in at least one patient, in combination with ZMapp and a white blood cell transfusion from an Ebola survivor, during the last Ebola outbreak. But efficacy experience remains anecdotal. Favipiravir is a broad-spectrum RNA polymerase inhibitor. It is also in phase III trials for treatment of influenza.

In May, a scientific advisory panel to the WHO noted that ZMapp had "the highest-quality data" for use in this emergency setting. They also prioritized the use of remdesivir over REGN-EB3 and favipiravir where possible. The said more data was needed before they could recommend mAb114 (see Nature Reviews Drug Discovery for more details on these drugs).

The WHO and Merck \& Co. are also testing the prophylactic efficacy of Ebola vaccine rVSV-ZEBOV in the DRC. rVSV-ZEBOV consists of a recombinant vesicular stomatitis virus that expresses the Zaire Ebolavirus glycoprotein. Researchers reported in The Lancet in 2017 that the vaccine offered substantial protection in a trial in around 4,000 at-risk individuals in the last outbreak. No cases of infection were found among vaccinated individuals from day 10 after vaccination. Asher Mullard 